\title{
Bedside Ultrasound-Guided Peripherally Inserted Central Catheter Placement by Critical Care Fellows in Critically Ill Patients: A Feasibility and Safety Study
}

\author{
Jeeyoun Lim a, Chi Ryang Chung b, Jeong-Am Ryu b, Eunmi Gil a,b,c,* \\ ${ }^{a}$ Department of Surgery, Samsung Medical Center, Sungkyunkwan University School of Medicine, Seoul, Korea \\ ${ }^{b}$ Department of Critical Care Medicine, Samsung Medical Center, Sungkyunkwan University School of Medicine, Seoul, Korea \\ ${ }^{c}$ Department of Surgery, College of Medicine, Kangwon National University, Chuncheon, Gangwon, Kore
}

\section{Article history:}

Received: September 23, 2020

Revised: October 12, 2020

Accepted: January 29, 2021

\section{*Corresponding Author:}

\section{Eunmi Gil}

Department of Critical Care Medicine and Surgery, Samsung Medical Center, Sungkyunkwan University School of

Medicine, 81 Irwon-ro, Gangnam-gu, Seoul,

135-710, Korea

E-mail: eunmi.gil@samsung.com

\section{ORCID}

Jeeyoun Lim

https://orcid.org/0000-0002-7005-8465

Chi Ryang Chung

https://orcid.org/0000-0003-1830-307X

Jeong-Am Ryu

https://orcid.org/0000-0003-1705-848X

Eunmi Gil

https://orcid.org/0000-0002-2002-0936

\section{ABSTRACT}

Purpose: In the intensive care unit, a peripherally inserted central catheter (PICC) may be an alternative option to standard central venous catheters, particularly in patients with coagulopathies or at high risk of infection. The purpose of this research was to assess the feasibility of bedside ultrasound (US)-guided PICC placement by critical care fellows on intensive care units.

Methods: All bedside US-PICCs inserted by critical care fellows from July 2013 to September 2015 were retrospectively reviewed focusing on the rate of successful insertion, complications of insertion, or during maintenance.

Results: A total of 177 US-guided PICCs were inserted in 163 patients and included in the analysis. The median age was 62 years (IQR 50-70 years) and 104 cases (58.8\%) were male. There were 172 cases $(90.4 \%)$ of PICCs inserted in the upper arm. Anticoagulant therapy was used in 26 patients $(14.7 \%)$ and 8 patients $(5.2 \%)$ had severe coagulopathies. The median procedural time was 30 minutes (IQR 19-45 minutes). Insertion success rate was $93.2 \%$, and there were no major complications during insertions except for malposition (12.1\%). Catheters remained in place for a total of 3,878 days (median 16 days: IQR 8 -31 days). There was only 1 case $(0.6 \%)$ of catheter-related bloodstream infection, and 2 cases (1.2\%) of symptomatic venous thromboembolism.

Conclusion: Bedside US-guided placement of PICCs by critical care fellows is safe and feasible. The success rate of the procedure was "acceptable," and was not associated with significant risks of infectious and non-infectious complications, even in patients with coagulopathies.

Keywords: catheters, critical illness, ultrasound

\section{Introduction}

The use of a peripherally inserted central catheter (PICC) has many advantages in the intensive care unit (ICU) setting because insertion of this device is low risk, even in patients with altered coagulation and/or difficult neck anatomy [1]. PICC insertion can be carried out with no risk of pleurapulmonary damage and with no clinically significant risk of a local hemorrhage or hematoma, if compared with insertion of a standard central venous catheter (CVC) [2]. PICCs are usually considered as a device at low risk of catheter-related bloodstream infection (CRBSI), which may be an additional advantage in acutely ill patients [2].

From the early 1990s, PICCs have been used in clinical practice and the majority of placement procedures have been performed by interventional radiologists with the assistance of either contrast venography or fluoroscopy. However, the incidence of adverse events in critically ill patients remains high and constitutes a significant risk during intra-hospital transport [3,4], so if possible, PICC should be performed at the bedside for patients in the ICU. Nowadays in many countries, most of the PICCs are placed by a team of trained Grade IV nurses at the patient's bedside, usually in an outpatient setting, and not in an emergency situation [5,6]. In 2001, a 
study reported nationwide guidelines for preventing infections in hospitals, and determined an "acceptable outcome" for PICC placement by intensivists which were performed at the bedside in ICUs [7].

Given the paucity of contemporary data on PICC placement specifically by critical care fellows in the ICU, a retrospective cohort review of the experience regarding bedside ultrasound (US)-guided PICC placement by critical care fellows in ICU patients was performed.

\section{Materials and Methods}

\section{Patient selection}

All bedside US-guided PICCs inserted by intensivists in patients admitted to ICU at the Samsung Medical Center (1,961bed, university-affiliated, tertiary referral hospital in Seoul, Korea), from July 2013 to September 2015 were retrospectively reviewed. PICC were inserted in patients who were recovering from their acute illness but who still required venous access for $>2$ weeks. According to hospital polices, indications for the use of PICC were: (1) need for a central line for non-peripherally compatible infusates (parenteral nutrition, and/or infusion of drugs that require a central line $(\mathrm{pH}<5$ or $>9$, osmolarity $>$ $500 \mathrm{mOsm} / \mathrm{L}$, drugs associated with endothelial damage), and/ or: (2) need for infusion of peripherally compatible infusate $>2$ weeks, and/or: (3) need for patients with difficult venous access, and/or: (4) need for frequent blood sampling ( $>3$ times/day). In some cases, in acutely ill patients, when a CVC insertion was not appropriate or a PICC placement was felt necessary by the critical care fellows, PICCs were used.

\section{Procedures}

All procedures were performed by skilled critical care fellows who had previously inserted $>50$ cases of CVCs. Prior to the clinical use of PICC, fellows followed a bedside US-guided PICC training program. The training component consisted of didactic lecture about basic concept and information of PICC and a skills laboratory. The skills laboratory was performed in the Division of education and human resources. The Clinical simulation team was at the Samsung Medical Center and used vascular access gel phantoms. After training was completed, fellows entered a competency period that required 5 successful US-guided PICCs to be placed under direct supervision of an experienced operator.

The type of PICCs selected by critical care fellows was dependent on the patient's condition. A Scale 5 French DualLumen Turbo-Ject Power-Injectable PICC (Cook, Bloomington, MN, USA) or Scale 6 French Dual-Lumen Vaxcel PICC with PASV Valve (Navilyst, Marlborough, MA, USA) was used.
All PICCs were inserted using real-time ultrasound to guide the puncture as recommended by current UK guidelines [8], using a modified Seldinger technique with a micro-introducer. A $10-13 \mathrm{MHz}$ linear US probe. Maximal barrier precautions were consistently used during the procedure (cap, mask, sterile gown, sterile gloves, and vast sterile field). The critical care fellows measured the anteroposterior diameter of the relevant vein (basilic, cephalic or brachial vein) at the insertion point. Veins with a diameter $\geq 5 \mathrm{~mm}$ were considered suitable for the scale 5 French catheters, and veins $\geq 6 \mathrm{~mm}$ suitable for the scale 6 French catheters. The vein of first choice was the right upper-mid arm basilic vein. If the right basilic vein was too small for the size of the intended catheter, the right upper mid-arm cephalic vein was examined. If the right basilic or cephalic vein was not compressible for the US assessment, the left arm was examined. The protocol stipulated that if the right basilic or cephalic vein were not appropriated, veins in the left arm were examined starting with the basilic vein and then the cephalic vein. The brachial veins were used as a last choice. Other standard contraindications to PICC insertion, were small veins of the upper arm $(<5 \mathrm{~mm})$, local contraindications due to specific arm conditions (skin infection, burns, bruised or corded venous segment, recent DVT (< 30 days), paralytic arm, defibrillator or pacemaker insertion site in the arm, and previous axillary node dissection) as well as actual or impending chronic renal failure requiring an arteriovenous fistula. The catheter length was measured from the puncture point to the ipsilateral sternoclavicular joint and to the right 3rd intercostal space. A post-procedural chest $\mathrm{X}$-ray for checking the tip position was required to verify the correct position of the tip of the catheter. The tip position was documented using a fraction of the total length of the superior vena cava [SVC (innominate junction to atriocaval junction)] [9]. PICC placement was considered "optimal" if the tip of the catheter was located at the atriocaval junction and "acceptable" if located anywhere in the SVC. All PICCs were secured to the skin using suture less devices, as currently recommended [10]. Maintenance of the line and dressings were carried out according to the intervention bundle of the hospital policy (use of $2 \%$ chlorhexidine for antisepsis of the exit site, preferential use of transparent dressing, and change the dressings every 7 days) [10].

\section{Definition}

A diagnosis of CRBSI was made when the same organism was recovered from peripheral blood culture, and from direct culture of the catheter tip, or by the differential time to positivity method (a positive blood culture from the catheter $\leq 120$ minutes prior to a positive peripheral blood culture). Diagnosis of catheter-related thrombosis was determined by 
US examination (compression ultrasonography and duplex Doppler), performed only when clinically indicated by signs and symptoms suggesting venous occlusion (such as edema of the arm, PICC malfunction, unexplained local pain). Catheterrelated central venous thrombosis was defined as the presence (to any extent) of thrombosis in the subclavian vein (SCV), internal jugular vein, or brachiocephalic vein or in the superior vena cava on the side of PICC insertion.

\section{Data collection and statistics}

All relevant data was collected and information, including the patient's age and disease, indication for PICC insertion, Sequential Organ Failure Assessment (SOFA) score on the insertion day, procedure time, duration of the dwell time for the device, incidence of complications at insertion or during maintenance, and cause of removal. All data were analyzed by standard descriptive statistics. Values were reported as the median with IQR.

Table 1. Baseline characteristics.

\begin{tabular}{lc}
\hline \multicolumn{1}{c}{ Variable } & Total $(N=172)$ \\
\hline Age $(\mathrm{y})$ & $63.0(50.25-70.75)$ \\
\hline Gender, male & $102(59.3)$ \\
BMI & $22.2(19.37-25.27)$ \\
\hline SOFA score & $8.0(5.00-11.00)$ \\
\hline Medical ICU & $118(68.6)$ \\
\hline Surgical ICU & $54(31.4)$ \\
\hline ICU length of stay $(\mathrm{d})$ & $15.0(8.00-26.50)$ \\
\hline Platelet count $\times 103 / \mu \mathrm{L}$ & $113.0(65.00-208.00)$ \\
\hline Platelet count $\leq 2 \times 103 / \mu \mathrm{L}$ & $11(6.4)$ \\
\hline PT-INR & $1.2(1.08-1.49)$ \\
\hline PT-INR $\geq 2$ & $17(9.9)$ \\
\hline aPTT $(s)$ & $43.7(37.00-55.10)$ \\
\hline Therapeutic anticoagulation & $25(14.5)$ \\
\hline CRRT & $57(33.1)$ \\
\hline Mechanical ventilation & $104(60.5)$ \\
\hline Tracheostomy & $75(43.6)$ \\
\hline ECMO & $16(9.3)$ \\
\hline Vasopressor & $62(36.0)$ \\
\hline Pata & \\
\hline
\end{tabular}

Data are presented as $n$ (\%) or $n$ (median, IQR).

ICU= intensive care unit; SOFA= sequential organ failure assessment; $\mathrm{BMI}=$ body mass index; PT-INR= prothrombin time - international normalized ratio; aPTT= activated partial thromboplastin time; CRRT= continuous renal replacement therapy; ECMO= extracorporeal membrane oxygenator.

\section{Results}

\section{Patients' characteristics}

During the study period, 172 PICCs in 159 patients were inserted in the ICU by 17 critical care fellows. The median age was 63 years (IQR 50.25-70.75 years) and 102 cases (59.3\%) were male. The median ICU length of stay was 15 days (IQR 8-26.5 days) and the median SOFA score was 8 (IQR 5-11). Therapeutic anticoagulation was performed in 25 patients (14.5\%) during the procedure. There were 11 (6.4\%) patients with thrombocytopenia $<2,000$ platelets $/ \mu \mathrm{L}$ and 17 patients (9.9\%) had a prolonged prothrombin time international normalized ratio $>2$. Amongst them, 7 patients $(4.1 \%)$ had severe coagulopathies ( 2 patients with thrombocytopenia $<10,000$ platelets $/ \mu \mathrm{L}$ and 5 patients with a prolonged prothrombin time international normalized ratio $>5$ ). There were 75 patients (43.6\%) who had a tracheostomy and 16 patients (9.3\%) were supported by extracorporeal membrane oxygenation (Table 1 ).

\section{Procedural outcomes}

Difficult venous access (52.9\%) was the most common indication for the PICC placement. In 5 cases of active fluid resuscitation, PICCs were placed due to failed peripheral venous access and insertion of CVCs were inappropriate. Out of the 5 cases, 3 cases were due to severe coagulopathies and 2 cases were due to local conditions at the CVC insertion sites.

Table 2. Procedure outcomes.

\begin{tabular}{lc}
\hline Variable & Total $(n=172)$ \\
\hline Indications for PICC insertion & \\
Non-peripherally compatible infusates & $91(52.9)$ \\
Difficult venous access & $30.0(18.5-44.75)$ \\
Procedure time (min) & \\
Puncture site & $102(59.3)$ \\
Right upper arm & $62(36.0)$ \\
Left upper arm & $123(71.5)$ \\
Puncture vessel & $37(21.5)$ \\
Non-cephalic vein (basilic or brachial) & $155(90.1)$ \\
Cephalic vein & $160(93.0)$ \\
Power injectable PICC & $12(7.0)$ \\
Success rate & $10(5.8)$ \\
Failed attempt & $2(1.2)$ \\
\hline Puncture fail & \\
Remove due to malposition & \\
\hline
\end{tabular}

Data are presented as $n$ (\%) or $n$ (median, IQR).

$\mathrm{PICC}=$ peripherally inserted central catheter; $\mathrm{IQR}=$ interquartile range. 
The median procedure time was 30 minutes (IQR 18.5-44.75 minutes). The right upper arm (59.3\%) was the most commonly used access point. Most of the catheters were inserted via the basilic or brachial veins (71.5\%) and the cephalic vein cannulation rate was $22.4 \%$. Power-injectable PICCs were inserted in 155 cases (90.1\%) and the insertion success rate was 93.0\% (Table 2). Patient characteristics and procedures did not show statistically significant differences between success and failure cases. In case of primary malposition, the catheter was used so was included as a successful case. The median duration for catheter maintenance was 16 days (IQR 8-31 days) and most common cause of catheter removal was suspected CRBSI (27.3\%), however, CRBSI was not consequently diagnosed.

\section{Procedure related complications}

During the study period, there were no significant complications such as perforation of the intracranial veins, perforation of central veins, arrhythmias requiring intervention, hemorrhage or hematoma which requires transfusion, or arterial injury. The only immediate post procedural complication was malposition in 20 cases (12.5\%; Table 3). Although the SVC/right atrium junction was the preferred site for the catheter tip placement, out of 20 cases of malpositioned catheters, 17 catheters placed for simple infusion of intravenous fluid due to poor peripheral veins were repositioned at the axillary vein by pulling back the catheters.

Catheters remained in place a total of 3,810 days (median 16 days: IQR, 8-31 days). There was 1 case in the ICU of CRBSI where a PICC remained in place for 58 days. Extend-spectrum beta-lactamase Klebsiella pneumoniae was cultured from both peripheral and central blood cultures with positive
Table 3. Procedure outcome, immediate (success case).

\begin{tabular}{lc}
\hline \multicolumn{1}{c}{ Variable } & Total $(n=160)$ \\
\hline Primary malposition & $20(12.5)$ \\
Ipsilateral IJV & $10(6.3)$ \\
Ipsilateral SCV & $10(6.3)$ \\
Catheter tip location & \\
RA or SVC lower $1 / 3$ & $70(40.7)$ \\
SVC mid $1 / 3$ & $58(33.7)$ \\
SVC upper $1 / 3$ & $15(8.7)$ \\
Axillary vein & $17(9.9)$ \\
\hline
\end{tabular}

Data are presented as $n(\%)$.

$\mathrm{IJV}=$ internal jugular vein; $\mathrm{SCV}=$ subclavian vein; $\mathrm{RA}=$ right atrium; SVC= superior vena cava.

time sensitivity, and the same organism was grown in the catheter tip culture. Symptomatic catheter-related central venous thrombosis occurred in 2 patients in the ICU. A patient with an arm swelling revealed SCV thrombosis, and another patient who showed sudden desaturation revealed SCV to SVC thrombosis with pulmonary embolism. PICCs were removed after thrombosis was diagnosed, and patients were treated with anticoagulants. There were 7 cases of catheter removal due to malfunction and 10 cases of dislodgements or accidental removals of the device (Table 4). There was no significant differences in outcomes between the indications for placement of PICCs.

Table 4. Procedure outcomes, long-term (success case).

\begin{tabular}{|c|c|c|c|c|}
\hline \multirow{3}{*}{ Variable } & \multirow{3}{*}{ Total $(n=160)$} & \multicolumn{3}{|c|}{ Indications for PICC insertion } \\
\hline & & Non-peripherally compatible infusates & Difficult venous access & $p$ \\
\hline & & $(n=78)$ & $(n=82)$ & \\
\hline Catheter indwelling time (d) & $16.0(8.00-31.00)$ & $17.5(9.00-35.25)$ & $14.5(7.00-26.75)$ & 0.195 \\
\hline Total catheter days & 3,810 & 2,086 & 1,724 & \\
\hline Complications & & & & 0.550 \\
\hline CRBSI & 1 (0.6; $0.26 / 1,000$ catheter-days) & $1(1.3)$ & 0 & \\
\hline Symptomatic DVT & $2(1.3 ; 0.52 / 1,000$ catheter-days $)$ & 0 & $2(2.4)$ & \\
\hline Malfunction & $7(4.4)$ & $4(5.1)$ & $3(3.7)$ & \\
\hline Accidental removal or dislodgement & $10(6.3)$ & $6(7.7)$ & $4(4.9)$ & \\
\hline
\end{tabular}

Data are presented as $n$ (\%) or $n$ (median, IQR).

PICC = peripherally inserted central catheter; $I Q R=$ interqurtile range; CRBSI= catheter related blood stream infection; DVT= deep vein thrombosis. 


\section{Discussion}

There were a total of 177 PICC which were inserted by critical care fellows. The patients' median age was 62 years old, median SOFA score was 8 , and median ICU length of stay was 15 days. These results show a high success rate (93.2\%) of PICC placement, and there were no major complications. Even though the malposition rate after PICC placement was $12.1 \%$, many cases ( $n=17$ ) of malposition could be repositioned simply by withdrawal to make the catheter midline. During the PICC indwelling time long term complications were rare [CRBSI $n=1(0.6 \%, 0.26 / 1,000$ catheter-days $)$ and symptomatic DVT $n=2(1.2 \%, 0.52 / 1,000$ catheter-days $)]$.

The first clinical experience with PICCs in an ICU was reported in 1996 [11]. After many potential advantages of PICCs in the ICU have been reported, especially the low risk of central line-associated bloodstream infections, the applications for PICCs in critical care setting have increased. However, studies of bedside US-guided PICC insertion by critical care fellows in the ICU are scarce. To our knowledge, this study is the first clinical study reporting a detailed analysis of the complications associated with the use of bedside US-guided PICCs by critical care fellows in the ICU patients.

The PICC placement is a relatively simple procedure. However, in critically ill patients, intra-hospital transport for example to the interventional radiology center could result in harm. In addition, the injection of iodinated contrast medium could worsen renal insufficiency. Consequently, most critically ill patients who need central lines for $>2$ weeks maintain CVCs, and frequently change the insertion site. Standard CVC insertion has a higher risk of immediate complication such as hemothorax, pneumothorax, or insertion site hematoma compared with PICC insertion and sometimes it could be lethal for critically ill patients [12]. Moreover, PICC-associated central line-associated bloodstream infection rates were lower than those reported with traditional, non-tunneled CVCs [13]. This is probably because the exit site is less prone to contamination (upper mid-arm skin is characterized by a lower bacterial colonization if compared with the skin on the neck or in the infra-clavicular area) and this benefit was particularly evident in patients with a tracheostomy [8]. The rate of immediate complication was $12.1 \%$, but only due to malposition rather than pleura-pulmonary damage and local hemorrhage or hematoma. In patients with severe coagulopathies, a PICC placement could prevent hemorrhagic complications and reduce unnecessary blood transfusions to correct coagulation abnormalities for line access. In the age when CVC insertion and bedside US are familiar, insertion of PICC by a critical care fellow has become technically feasible. Usually, PICCs are not indicated for unstable patients. However, the experience of critical care fellows suggests that in some cases, rapid access allowed bedside US-guided PICC placement to be performed when access for inserting a CVC was not possible during patient fluid resuscitation. In these cases of fluid resuscitation, power-injectable PICCs were inserted which could deliver a very high rate of infusates (3-5 mL/second) [14].

In case of ICU patients, PICC placement may be more difficult compared with outpatients because many of them had been treated with positive pressure ventilation, edematous extremities, and had very poor peripheral veins in case of chronic illness, and many of them could not hold a position. Consequently, the risk of failure or malposition was higher compared with non-ICU patients. In this study, failed attempt rate was $6.8 \%$ and the catheter tip malposition rate was $12.1 \%$ when a chest X-ray was performed to check PICC placement. Other studies show less than $3 \%$ of cases were due to malposition ensuring the catheter tip position with medical devices during PICC placement $[15,16]$. However, the study cohorts for these studies were outpatients. This was lower than a PICC study which showed $13 \%$ failure and $22.3 \%$ catheter tip malposition [5]. If the risk of intra-hospital transport of ICU patients is considered, the bedside US-PICC placement by a critical care fellow is quite useful in minimizing resources. Moreover, critical care fellows know their ICU patients well and could rapidly access equipment even in cases of acute illness.

Our study had several limitations. Firstly, there may be a potential risk of confounding variables because the study was not a randomized controlled trial. In addition, 1 physician placed $>1 / 3$ of PICCs in the study, and the success rate may not be repeated by multiple operators. Another limitation of the study was the blind technique of catheter tip location. The catheter tip position was verified with a post-procedural chest X-ray, without fluoroscopy or an electrocardiography. This results in a relatively high rate of malposition compared with previous studies using a real time catheter tip position. Considering the results of this preliminary retrospective study, further prospective, controlled, randomized trials comparing the clinical outcome of CVCs with PICCs in ICU patients are warranted. In addition, consideration should be given to the establishment of procedural training in PICC placement for critical care fellows.

\section{Conclusion}

This preliminary retrospective study showed that bedside US-guided PICC placement by critical care fellows is safe and feasible in most ICU patients, even in patients with severe coagulopathies and those requiring fluid resuscitation. There were no major immediate complications except for malposition, and CRBSI, and symptomatic thrombosis was uncommon. Bedside US-guided PICC placement by critical 
care fellows could be considered in ICU patients who were recovering from their acute illness, but who still required venous access for $>2$ weeks, and could be considered in some cases of acute illness.

\section{Conflicts of Interest}

The authors have no conflicts of interest to declare.

\section{Supplementary Material}

Supplementary Table S1 can be found in the online version at https://doi.org/10.17479/jacs.2021.1.30.

\section{References}

[1] Chopra V, Flanders SA, Saint S, Woller SC, O'Grady NP, Safdar N, et al. The Michigan Appropriateness Guide for Intravenous Catheters (MAGIC): Results From a Multispecialty Panel Using the RAND/UCLA Appropriateness Method. Ann Intern Med 2015;163(6 Suppl):S1-40.

[2] Pittiruti M, Hamilton H, Biffi R, MacFie J, Pertkiewicz M. ESPEN Guidelines on Parenteral Nutrition: Central venous catheters (access, care, diagnosis and therapy of complications). Clin Nutr 2009;28(4):365-77.

[3] Lahner D, Nikolic A, Marhofer P, Koinig H, Germann P, Weinstabl C, et al. Incidence of complications in intrahospital transport of critically ill patients: Experience in an Austrian university hospital. Wien Klin Wochenschr 2007;119(13-14):412-6.
[4] Papson JP, Russell KL, Taylor DM. Unexpected events during the intrahospital transport of critically ill patients. Acad Emerg Med 2007;14(6):574-7.

[5] Neuman ML, Murphy BD, Rosen MP. Bedside placement of peripherally inserted central catheters: A cost-effectiveness analysis. Radiology 1998;206(2):423-8.

[6] Sainathan S, Hempstead M, Andaz S. A single institution experience of seven hundred consecutively placed peripherally inserted central venous catheters. J Vasc Access 2014;15(6):498-502.

[7] Lee D, Park C. Clinical Feasibility of Ultrasound Guided Placement of Peripherally Inserted Central Catheters by Intensivist: Preliminary Report. J Acute Care Surg 2014;4(1):13-7.

[8] Pratt RJ, Pellowe CM, Wilson JA, Loveday HP, Harper PJ, Jones SR, et al. epic2: National evidence-based guidelines for preventing healthcareassociated infections in NHS hospitals in England. J Hosp Infect 2007;65 Suppl 1:S1-64.

[9] Caers J, Fontaine C, Vinh-Hung V, De Mey J, Ponnet G, Oost C, et al. Catheter tip position as a risk factor for thrombosis associated with the use of subcutaneous infusion ports. Support Care Cancer 2005;13(5):32531.

[10] O'Grady NP, Alexander M, Burns LA, Dellinger EP, Garland J, Heard SO, et al. Guidelines for the prevention of intravascular catheter-related infections. Clin Infect Dis 2011;52(9):e162-93.

[11] Ng PK, Ault MJ, Maldonado LS. Peripherally inserted central catheters in the intensive care unit. J Intensive Care Med 1996;11(1):49-54.

[12] Loughran SC, Borzatta M. Peripherally inserted central catheters: A report of 2506 catheter days. JPEN J Parenter Enteral Nutr 1995;19(2):133-6.

[13] Maki DG, Kluger DM, Crnich CJ. The risk of bloodstream infection in adults with different intravascular devices: A systematic review of 200 published prospective studies. Mayo Clin Proc 2006;81(9):1159-71.

[14] Pittiruti M, Brutti A, Celentano D, Pomponi M, Biasucci DG, Annetta MG, et al. Clinical experience with power-injectable PICCs in intensive care patients. Crit Care 2012;16(1):R21.

[15] Pittiruti M, La Greca A, Scoppettuolo G. The electrocardiographic method for positioning the tip of central venous catheters. J Vasc Access $2011 ; 12(4): 280-91$.

[16] Lelkes V, Kumar A, Shukla PA, Contractor S, Rutan T. Analysis of the Sherlock II tip location system for inserting peripherally inserted central venous catheters. Clin Imaging 2013;37(5):917-21. 